\title{
Monte-Carlo simulation of the memory effect in random media beyond the diffusion limit
}

\author{
Chen Bar ${ }^{\mathrm{a}}$, Marina Alterman ${ }^{\mathrm{a}}$, Ioannis Gkioulekas ${ }^{\mathrm{b}}$, and Anat Levin ${ }^{\mathrm{a}}$ \\ ${ }^{a}$ Department of Electrical Engineering, Technion, Haifa, Israel. \\ ${ }^{\mathrm{b}}$ Robotics Institute, Carnegie Mellon University, Pittsburgh, PA, USA.
}

\begin{abstract}
We derive a physically accurate and computationally efficient Monte Carlo algorithm that can be used to evaluate the complex statistics of speckle fields in scattering media. This allows evaluating and studying second-order speckle statistics, such as the memory effect, for a large variety of material and imaging parameters, including turbid materials. This helps bridge the gap between analytical formulas, derived under restrictive assumptions such as diffusion, and empirical lab measurements. It also opens up the possibility for discovering new types of correlation effects, and using those to improve our ability to see through and focus into random media.
\end{abstract}

Keywords: Speckle statistics, Memory effect, Monte Carlo.

\section{INTRODUCTION}

Coherent waves propagating through random media lead to high fluctuation patterns known as speckles. These noise-like patterns are characterized by strong statistical properties, such as the so-called memory effect (ME), which are at the core of imaging techniques for applications as diverse as tissue imaging, motion tracking, and seeing around the corner. ${ }^{1}$ The memory effect allows seeing through scattering media ${ }^{1,2}$ in microscopic resolution, far beyond what can be achieved by incoherent diffusive techniques. Due to its wide applicability, there have been many efforts to study the extent of the memory effect for different materials. ${ }^{3}$ Available theoretical results only apply under restrictive assumptions, such as diffusion and the Fokker-Planck limit. Given the limited theory, there have been many experimental lab efforts ${ }^{4}$ measuring the range of the memory effect for tissue and other materials. The empirical empirical evidence from these experiments suggests that the angular range of the memory effect can, in practice, be order of magnitudes larger than the theoretical predictions derived under the diffusion approximation. We propose a computationally efficient Monte Carlo (MC) algorithm that can evaluate speckle statistics as a function of bulk material parameters, without the need of diffusion assumptions. The accuracy of our algorithm is verified against an exact wave solver; at the same time, our algorithm is orders of magnitude more efficient computationally. This enables the practical evaluation of the memory effect beyond the diffusion limit, and the ability to predict new types of speckle correlations that were previously unexplored.

\section{COMPARISON OF MONTE CARLO AND EXACT WAVE EVALUATION OF SPECKLE STATISTICS}

To define speckle covariance, we assume a set of microscopic locations of scatterers $O=\left\{\mathbf{o}_{1}, \mathbf{o}_{2}, \ldots\right\}$ inside a medium. This medium is illuminated from direction $\hat{\mathbf{i}}$, and imaged by a sensor at position $\hat{\mathbf{v}}$. We can then use a wave equation solver ${ }^{5}$ to solve the Helmholtz equation, and evaluate the resulting complex-valued speckle field $u_{\mathbf{v}}^{\mathbf{i}, O}$. By repeating this procedure for multiple random scatterer configurations $O^{n}$, sampled from the same density describing the medium, we can compute the speckle covariance:

$$
C_{\mathbf{v}_{1}, \mathbf{v}_{2}}^{\mathbf{i}_{1}, \mathbf{i}_{2}} \approx \frac{1}{N} \sum_{n=1}^{N} u_{\mathbf{v}_{1}}^{\mathbf{i}_{1}, O^{n}} \cdot u_{\mathbf{v}_{2}}^{\mathbf{i}_{2}, O^{n *}},
$$

Further author information:

Anat Levin: anat.levin@technion.ac.il, Telephone: +972-4-829-5921 
$\left(0^{\circ}, 0^{\circ}\right)$
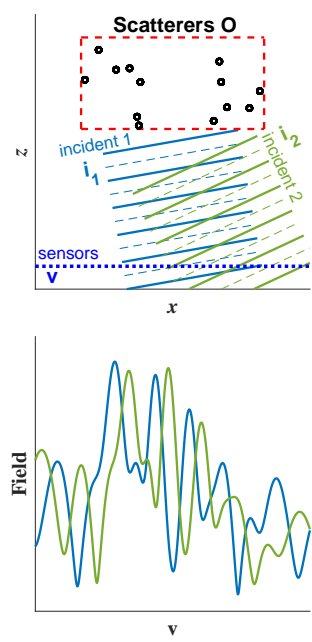

$\left(0^{\circ}, 1^{\circ}\right)$
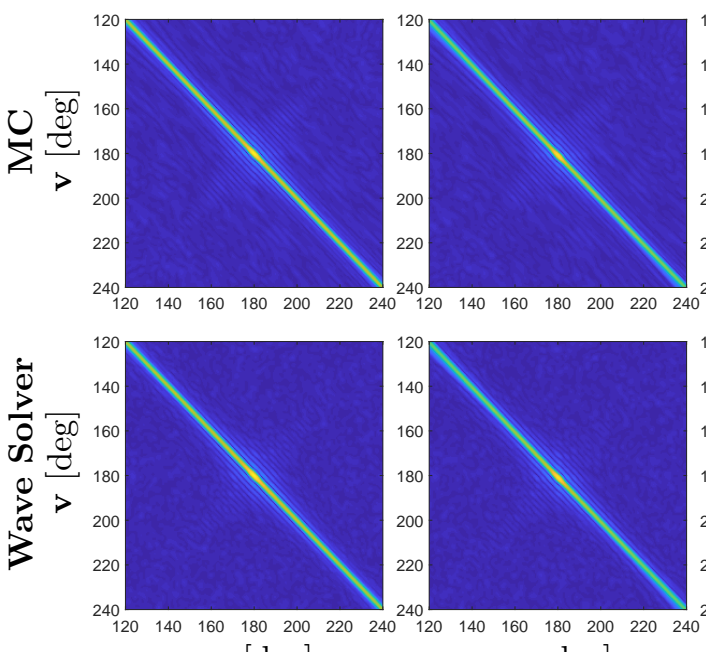

$\mathbf{v}[\operatorname{deg}]$

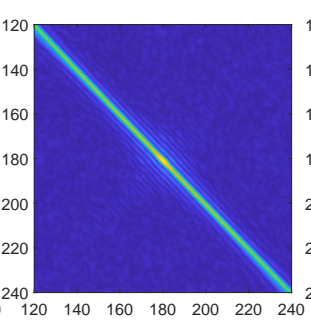

v deg] $\left(0^{\circ}, 4^{\circ}\right)$
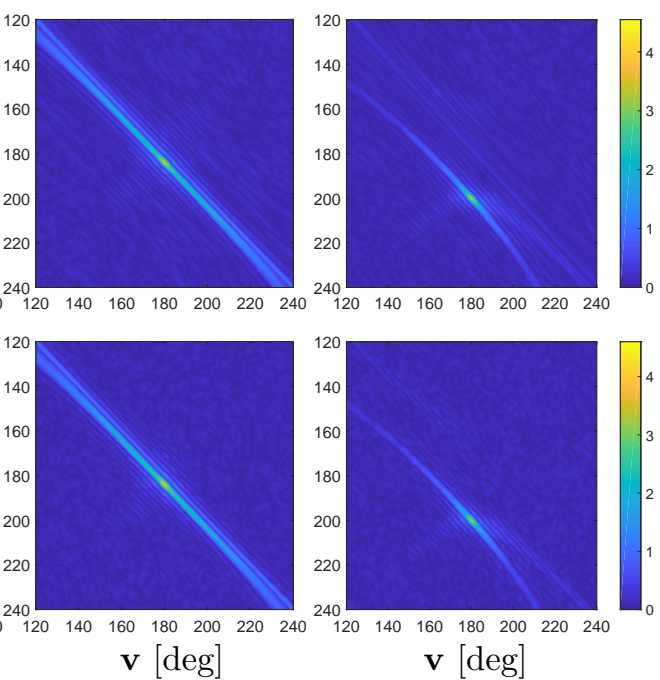

Figure 1: Setup and validation: The left part demonstrates a setup of a 2D target illuminated by two plane waves and sensors measuring the backscattered field. Below it, a typical pair of fields generated by two different illumination directions: the blue and green fields are shifted versions of each other, demonstrating the memory effect. On the right, our MC covariances compared against a "ground truth" covariance obtained from a wave solver. We simulate a sample of size $20 \lambda \times 20 \lambda$ with a mean free path of $10 \lambda$. Each of the 4 sets of covariance matrices represents statistics of fields generated by a different pair of illumination directions.

where $u_{\mathbf{v}_{1}}^{\mathbf{i}_{1}, O^{n}}, u_{\mathbf{v}_{2}}^{\mathbf{i}_{2}, O^{n}}$ are two waves, scattered by the same particle set when illuminated from two different directions. The wave solver is computationally costly and does not scale to scenes with more than a few hundred particles. We suggest an MC algorithm for the evaluation of the covariance $C_{\mathbf{v}_{1}, \mathbf{v}_{2}}^{\mathbf{i}_{1}, \mathbf{i}_{2}}$, which is orders of magnitude faster and scales to much larger scenes. Our formulation starts from the theory of Twersky ${ }^{6}$ to derive a pathintegral expression for speckle covariance, which leads to a simple sampling algorithm for evaluating this integral.

In Fig. 1 we visualize covariance matrices obtained with the two approaches, demonstrating the accuracy of our results. The matrices also demonstrate the non trivial statistics of speckles. In particular, the covariance has a dominant diagonal that is offset from the center. This shift is the memory effect: two illumination directions propagating through the same set of particles lead to speckle fields that are highly-correlated and shifted versions of each other, as illustrated at the lower left plot of Fig. 1. When the angle difference is larger, the classical version of the memory effect no longer holds and the covariance is no longer a shifted diagonal. However, we can still observe some correlation along a curved set of viewing directions. To the best of our knowledge, such correlations have not yet been explored, and provide an exciting direction for future research, by pointing to ways for expanding the angular range of computational imaging applications relying on the memory effect.

\section{EVALUATING THE MEMORY EFFECT}

There have been many attempts to understand the exact extent under which the memory effect holds. The main theoretical result, derived under diffusion assumptions, states that the correlation between speckles produced by illumination directions with angle difference $\theta$ decays as $C(\theta)=(k \theta L)^{2} /(\sinh (k \theta L))^{2}$. Empirical evidence suggests that, in practice, the decay is slower than the theory predicts, as long as the optical depth (that is, the average number of scattering events) is lower than the diffusion regime. This difference becomes more pronounced in volumes with high absorption, because absorption attenuates multiply-scattered paths, and especially in volums with a very forward-scattering phase function. Both of these cases are important in practice, as they match the scattering properties of tissue. Given the difficulty of predicting the memory effect in these situations, the angular extent of the memory effect for materials of interest is often measured empirically in the lab. ${ }^{4}$ 

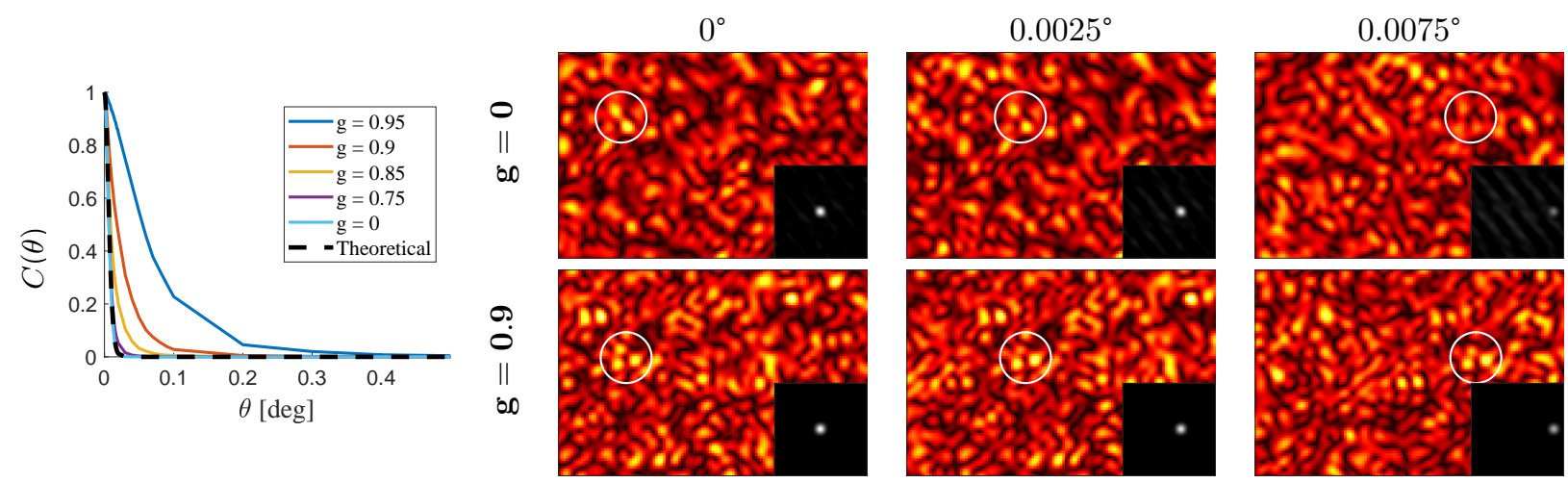

Figure 2: Memory effect evaluation: Left: speckle correlation $C(\theta)$ as a function of difference between illumination directions, for Henyey-Greenstein (HG) phase functions with different anisotropy parameters $g$. Right: speckle images rendered by our algorithm with two $g$ values, for a few illumination directions, as well as their auto-correlation (black insets). The speckle shift is evident, but as the angle difference increases, correlation decays and the decay rate is different for different material parameters.

Our MC algorithm can relax this experimental burden, as it allows for the numerical evaluation of $C(\theta)$ as a function of many material and imaging parameters. In Fig. 2, we use this approach to evaluate speckle correlations for a few HG phase functions with different anisotropy parameters $g$. In the isotropic case, $g=0$, our numerical results match theoretical predictions of diffusion theory, but as anisotropy increases the memory effect range can be order of magnitudes wider than theoritically expected.

A variant of the same MC formulation allows rendering speckle images that are physically-correct, as they capture all first-order and second-order statistics within a single speckle image and across multiple illuminations. In Fig. 2, we show examples of such speckle images: As expected from the memory effect, they are consistent under small changes in illumination angle, and their correlation gradually decays as the illumination difference increases. The correlation decay is faster for lower $g$ values, in agreement with the correlation curves in Fig. 2.

\section{DISCUSSION AND FUTURE APPLICATIONS}

We suggest a computationally efficient algorithm for the evaluation of speckle statistics. The result is physically accurate, as it closely matches solutions of the wave equation, and reproduces known physical phenomena such as memory effect and coherent backscattering. Better modeling of speckle statistics and the discovery of new types of correlations is promising for seeing through and focusing into random media. The ability to synthesize physically-correct speckle data can additionally facilitate the incorporation of machine learning algorithms in applications where the collection of large-scale training data is impossible.

Acknowladgment: This work was supported by ERC 635537, ISF 1046-14, Ollendorff Minerva Center of the Technion, DARPA REVEAL HR0011-16-C-0028 and NSF Expeditions CCF-1730147.

\section{REFERENCES}

[1] Batarseh, M., Sukhov, S., Shen, Z., Gemar, H., Rezvani, R., and Dogariu, A., "Passive sensing around the corner using spatial coherence," Nature Communications (2018).

[2] Katz, O., Heidmann, P., Fink, M., and Gigan, S., "Non-invasive single-shot imaging through scattering layers and around corners via speckle correlation," Nat. Photonics (2014).

[3] Osnabrugge, G., Horstmeyer, R., Papadopoulos, I. N., Judkewitz, B., and Vellekoop, I. M., "Generalized optical memory effect," Optica (2017).

[4] Schott, Bertolotti, Léger, Bourdieu, and Gigan, "Characterization of the angular memory effect of scattered light in biological tissues," Opt. Express (2015). 
[5] Thierry, B., Antoine, X., Chniti, C., and Alzubaidi, H., " $\mu$-diff: An open-source matlab toolbox for computing multiple scattering problems by disks," Computer Physics Communications 192, 348 - 362 (2015).

[6] Twersky, V., "On propagation in random media of discrete scatterers," Am. Math. Sot. Symp. Stochastic Processes in Mathematical Physics and Engineering, Vol. 16, p. 84 (1964). 